\title{
Buckling and post-buckling behaviour of shallow - nearly flat cylindrical panels under axial compression
}

\author{
E. MAGNUCKA-BLANDZI*1 and K. MAGNUCKI ${ }^{2}$ \\ ${ }^{1}$ Institute of Mathematics, Poznan University of Technology, ul. Piotrowo 3A, 60-965 Poznań, Poland \\ ${ }^{2}$ Institute of Applied Mechanics, Poznan University of Technology, ul. Jana Pawla II 24, 60-965 Poznań, Poland
}

\begin{abstract}
The paper is devoted to buckling problem of axially compressed shallow cylindrical panels. Governing differential equations of the nonlinear theory of shallow cylindrical shells are analytically solved. Critical stresses and equilibrium paths of the panels with small curvatures are analytically studied. The formula of the critical stresses for almost flat, cylindrical panels is derived. The "shallowness" of the panel is given by the parameter $\alpha$ and formulae are derived for a range of this parameter. The range of values of sectorial angle for these panels is also defined.
\end{abstract}

Key words: rectangular plate, cylindrical shell, elastic buckling, equilibrium paths.

\section{Introduction}

The buckling and post buckling problems are investigated from more than hundred years. Results of the theoretical, numerical and experimental investigations were elaborated in many papers and monographs, selected, for example, in chronological order: Timoshenko and Gere [1], Hutchinson and Koiter [2], Budiansky [3], Brush and Almorth [4], Donnell [5], Grigoluk and Kabanov [6], Yamaki [7], Bushnell [8], Simitses [9], Bažant and Cedolin [10], Hunt and Neto [11], Teng [12], Lord et al. [13], Ventsel and Krauthammer [14], Rotter [15], Luong and Tri [16]. The problem of a lower estimation of critical stress of axially compressed shallow cylindrical panels was presented by Magnucki [17]. Buckling problems of plates and shells are at present studied. Dębowski et al. [18] theoretically investigated the dynamic stability of a metal foam rectangular plate, however Belica et al. [19] studied the dynamic stability of an isotropic metal foam cylindrical shell subjected to external pressure and axial compression. Moulton and Goriely [20] presented interesting problem of circumferential buckling instability of a cylindrical elastic tube under uniform radial external pressure. Polat nad Calayir [21] studied geometrically nonlinear static and dynamic response of shells of revolution. Magnucki and Jasion [22] analytically described the pre-buckling, buckling and post-buckling states of barrelled shells under pressure.

Koiter [23] indicated to the buckling problem of shallow cylindrical panels. Then Thompson and Hunt [24] briefly characterized the problem. Lancaster et al. [25] emphasized the sensitivity of the buckling load to the presence of initial imperfections.

The subject of the paper is a family of axially compressed shallow cylindrical panels with four edges simply supported (Fig. 1). Critical stresses and equilibrium paths of the panels are analytically calculated. A rectangular plate as a flat panel

\footnotetext{
*e-mail: ewa.magnucka-blandzi@put.poznan.pl
}

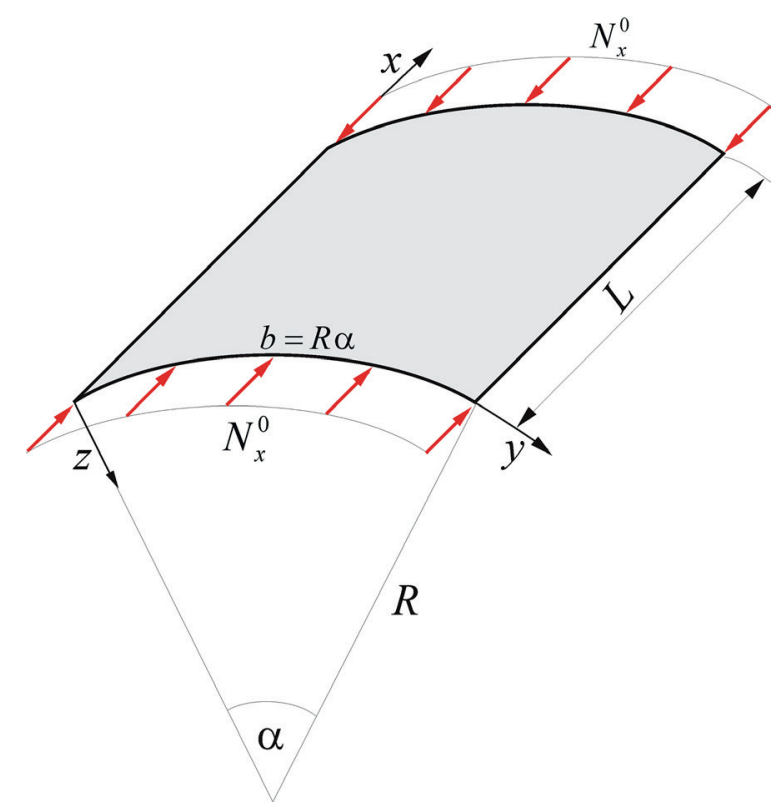

Fig. 1. Axially compressed shallow-almost flat cylindrical panel

is a particular structure of this family. The analytic formula of the critical stresses for almost flat, cylindrical panels is presented.

The main goal of the study consists in analytical formulation of the critical and post critical states for the family of shallow - almost flat cylindrical panels with consideration of the flat panel.

\section{Buckling problem of the shallow cylindrical panels}

2.1. Analytical solution of the governing equations. The governing differential equations of the nonlinear theory of shallow 
cylindrical shells called the von $\mathrm{K} \backslash$ 'arm \'an-Donnell equations are in the following form

$$
\begin{aligned}
& D \nabla^{4} w=\frac{1}{R} \frac{\partial^{2} F}{\partial x^{2}}+\Re(w, F), \\
& \nabla^{4} F+E t\left[\frac{1}{R} \frac{\partial^{2} w}{\partial x^{2}}+\frac{1}{2} \Re(w, w)\right]=0
\end{aligned}
$$

where:

$$
\begin{array}{ll}
\mathrm{D}=E t^{3} /\left[12\left(1-\nu^{2}\right)\right] & - \text { stiffness of bending, } \\
E, \nu & - \text { constants of material, } \\
t & - \text { thickness of the shell } \\
\Re(w, F)=\frac{\partial^{2} w}{\partial x^{2}} \frac{\partial^{2} F}{\partial y^{2}}- & 2 \frac{\partial^{2} w}{\partial x \partial y} \frac{\partial^{2} F}{\partial x \partial y}+\frac{\partial^{2} w}{\partial y^{2}} \frac{\partial^{2} F}{\partial x^{2}} \\
& - \text { nonlinear differential operator, } \\
w(x, y) & - \text { deflection of the panel, } \\
F(x, y) & - \text { force function (Airy function). }
\end{array}
$$

The in-plane forces - intensities:

$$
N_{x}=\frac{\partial^{2} F}{\partial y^{2}}, S_{x y}=-\frac{\partial^{2} F}{\partial x \partial y}, N_{y}=\frac{\partial^{2} F}{\partial x^{2}} .
$$

The buckling shape - the deflection of the panels is assumed in the form

$$
w(x, y)=w_{a} \sin \frac{m \pi x}{L} \sin \frac{n \pi y}{b},
$$

where

$$
\begin{aligned}
& b, L-\text { sizes of the panels (Fig. 1), } \\
& m, n-\text { natural numbers, } \\
& w_{\alpha} \quad-\text { amplitude of deflection. }
\end{aligned}
$$

Substituting the function (3) into second equation of the system (1), and taking into account the axial load, one obtains the force function in the following form

$$
F(x, y)=-\frac{1}{2} N_{x}^{o} y^{2}+\operatorname{Etf}_{F}(x, y)
$$

where

$$
\begin{aligned}
& f_{F}(x, y)=\frac{b^{2}}{\left[n \pi\left(k_{m n}+k_{n m}\right)\right]^{2}} \frac{w_{a}}{R} \sin \frac{m \pi x}{L} \sin \frac{n \pi y}{b}+ \\
& +\frac{w_{a}^{2}}{32} k_{n m}^{2} \cos \frac{2 m \pi x}{L}+\frac{w_{a}^{2}}{32} k_{m n}^{2} \cos \frac{2 n \pi y}{b}, k_{m n}=\frac{m}{n} \frac{b}{L}, \\
& k_{n m}=\frac{n}{m} \frac{L}{b} \text { are dimensionless parameters, } \\
& N_{x}^{0} \text { is the intensity of uniformly distributed axial } \\
& \quad \text { compression force. }
\end{aligned}
$$

The first equation of the system (1) is approximately solved with the Galerkin's method. The orthogonal condition

$$
\begin{gathered}
\int_{0}^{b} \int_{0}^{L}\left[D \nabla^{4} w-\frac{1}{R} \frac{\partial^{2} F}{\partial x^{2}}-\Re(w, F)\right] \\
\sin \frac{m \pi x}{L} \sin \frac{n \pi y}{b} d x d y=0
\end{gathered}
$$

gives

$$
\begin{aligned}
\frac{\sigma_{x}^{o}}{E}= & \frac{\left[n \pi\left(k_{m n}+k_{n m}\right) \frac{t}{b}\right]^{2}}{12\left(1-\nu^{2}\right)}+\frac{\left(\frac{b}{R}\right)^{2}}{\left[n \pi\left(k_{m n}+k_{n m}\right)\right]^{2}}- \\
& -k_{w 1} \frac{w_{a}}{R}+k_{w 2}\left(\frac{w_{a}}{b}\right)^{2},
\end{aligned}
$$

where

$$
\begin{array}{ll}
\sigma_{x}^{o}=\frac{N_{x}^{o}}{t} & - \text { stress, } \\
k_{w 1}=\frac{2}{3 \pi^{2}}\left[k_{n m}^{2}+\frac{16}{\left(k_{m n}+k_{n m}\right)^{2}}\right], & \\
k_{w 2}=\frac{\pi^{2}}{16}\left(k_{m n}^{2}+k_{n m}^{2}\right) \quad-\text { parameters. }
\end{array}
$$

The expression (6) is the base for analysis of buckling and post-buckling problems of shallow cylindrical shells.

2.2. Critical stresses. The critical state of the structures initiates the buckling process. Assuming $w_{\alpha}=0$ in the expression (6) one obtains the critical state

$$
\begin{aligned}
\frac{\sigma_{x}^{o}}{E} & =\frac{\left[n \pi\left(k_{m n}+k_{n m}\right)\right]^{2}}{12\left(1-\nu^{2}\right)}\left(\frac{t}{b}\right)^{2}+ \\
& +\frac{1}{\left[n \pi\left(k_{m n}+k_{n m}\right)\right]^{2}}\left(\frac{b}{R}\right)^{2}
\end{aligned}
$$

Length of the circle arc of the cylindrical panel $b=R \alpha$ is constant for the shallow shells family (Fig. 1). For some values of the radius $R=R_{s}$ and the angle $\alpha=\alpha_{s}$ the stress (7) is equal of the Lorenz-Timoshenko-Southwell critical stress

$$
\frac{\sigma_{C R}^{(L-T-S)}}{E}=\frac{1}{\sqrt{3\left(1-\nu^{2}\right)}} \frac{t}{R_{s}}
$$

therefore

$$
\begin{aligned}
& \frac{\left[n \pi\left(k_{m n}+k_{n m}\right)\right]^{2}}{12\left(1-\nu^{2}\right)}\left(\frac{t}{b}\right)^{2}+ \\
& +\frac{1}{\left[n \pi\left(k_{m n}+k_{n m}\right)\right]^{2}}\left(\frac{b}{R_{s}}\right)^{2}=\frac{1}{\sqrt{3\left(1-\nu^{2}\right)}} \frac{t}{R_{s}},
\end{aligned}
$$

or, after a simple transformation

$\left[\frac{n \pi\left(k_{m n}+k_{n m}\right)}{2 \sqrt{3\left(1-\nu^{2}\right)}} \frac{t}{b}-\frac{1}{n \pi\left(k_{m n}+k_{n m}\right)} \frac{b}{R_{s}}\right]^{2}=0$,

from which

$$
\frac{b}{R_{s}}=\alpha_{s}=\frac{\left[n \pi\left(k_{m n}+k_{n m}\right)\right]^{2}}{2 \sqrt{3\left(1-\nu^{2}\right)}} \frac{t}{b} .
$$

Minimum of this expression as a function occurs for $n=1$ and $k_{m n}=k_{n m}=1$, thus the boundary angle

$$
\alpha_{s}=\frac{2 \pi^{2}}{\sqrt{3\left(1-\nu^{2}\right)}} \frac{t}{b} .
$$

The range of the sectorial angle of the shallow shells family is as follows

$$
0 \leq \alpha \geq \alpha_{s}
$$




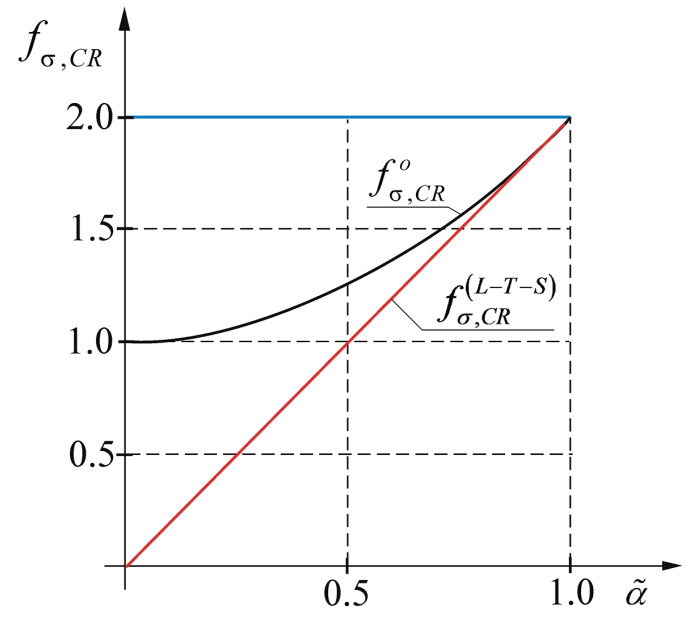

Fig. 2. Graphs of dimensionless functions of critical stresses

The critical stress $(7)$, for $n=1, k_{m n}=k_{n m}=1$, and with consideration of the expression (12) is as follows

$$
\frac{\sigma_{x, C R}^{o}}{E}=\frac{\pi^{2}}{3\left(1-\nu^{2}\right)}\left(\frac{t}{b}\right)^{2} f_{\sigma, C R}^{o},
$$

where

$$
f_{\sigma, C R}^{o}=1+\widetilde{\alpha}^{2}
$$

is a dimensionless function of the critical stress

$$
\widetilde{\alpha}=\frac{\alpha}{\alpha_{s}}
$$

is a relative sectorial angle.

The Lorenz-Timoshenko-Southwell critical stress (8) may be written as follows

$$
\frac{\sigma_{C R}^{(L-T-S)}}{E}=\frac{\pi^{2}}{3\left(1-\nu^{2}\right)}\left(\frac{t}{b}\right)^{2} f_{\sigma, C R}^{(L-T-S)},
$$

where

$$
f_{\sigma, C R}^{(L-T-S)}=2 \widetilde{\alpha}
$$

is a dimensionless function of the Lorenz-Timoshenko-Southwell critical stress.

The graphs of these dimensionless functions are shown in Fig. 2.

The expression (14) determines the critical stress for the family of shallow - almost flat cylindrical panels and the flat panel (the rectangular plate). If $\widetilde{\alpha}=0(R \rightarrow \infty)$ then the cylindrical panel becomes the rectangular plate, and the expression (14) determines well known in the literature a critical stress

$$
\frac{\sigma_{x, C R}^{o}}{E}=\frac{\pi^{2}}{3\left(1-\nu^{2}\right)}\left(\frac{t}{b}\right)^{2}
$$

for rectangular plates, because $f_{\sigma, C R}^{o}=1$.

The Lorenz-Timoshenko-Southwell formula (16) does not determine the critical stress for the family of almost flat cylindrical panels or the flat panel. Based on this formula for the flat panel the critical stress is equal zero.

2.3. Equilibrium paths. The post-critical state is described by the equilibrium path. The expression (6) for $n=1$, $k_{m n}=k_{n m}=1$, and with consideration of the expression (12) is in the following form

$$
\frac{\sigma_{x}^{o}}{E}=\frac{\pi^{2}}{3\left(1-\nu^{2}\right)}\left(\frac{t}{b}\right)^{2} f_{\sigma}^{o}\left(\widetilde{\alpha}, \widetilde{w}_{a}\right)
$$

where dimensionless function of the equilibrium path

$$
\begin{aligned}
f_{\sigma}^{0}\left(\widetilde{\alpha}, \widetilde{w}_{a}\right) & =1+(\widetilde{\alpha})^{2}-\frac{20}{3 \pi^{2}} \sqrt{3\left(1-\nu^{2}\right)} \widetilde{\alpha} \widetilde{w}_{a}+ \\
& +\frac{3}{8}\left(1-\nu^{2}\right) \widetilde{w}_{a}^{2}
\end{aligned}
$$

and $\widetilde{w}_{a}=w_{a} / t-$ dimensionless deflection.

The graphs of the function (19) for $\widetilde{\alpha}=0,0.5,1.0$ are shown in Fig. 3.

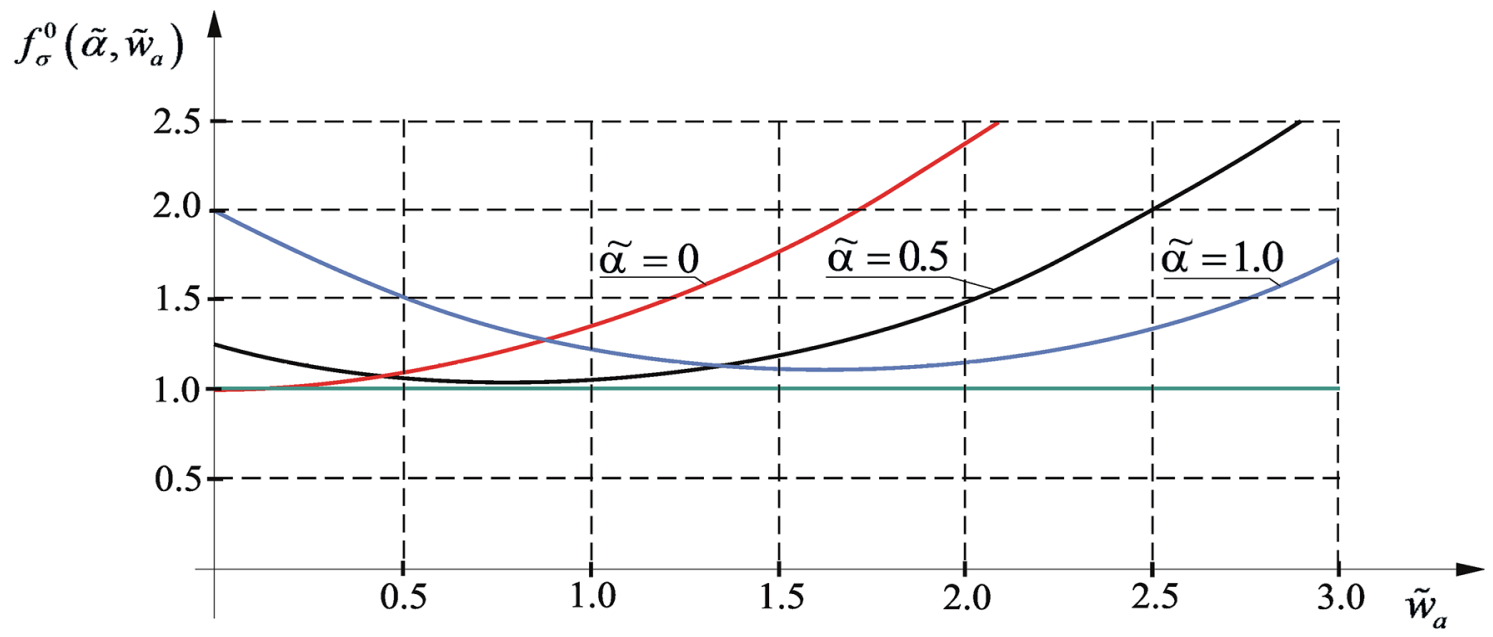

Fig. 3. Equilibrium paths of the flat panel and shallow cylindrical panels 
Minimum of the equilibrium paths (18)

$$
\min _{\widetilde{w}_{a}}\left\{\frac{\sigma_{x}^{o}}{E}\right\}=\frac{\pi^{2}}{3\left(1-\nu^{2}\right)}\left(\frac{t}{b}\right)^{2}\left[1+\left(1-\frac{800}{9 \pi^{4}}\right) \widetilde{\alpha}^{2}\right],
$$

and occurs for

$$
\widetilde{w}_{a}=\frac{80}{3 \pi^{2} \sqrt{3\left(1-\nu^{2}\right)}} \widetilde{\alpha}
$$

The minimum values of the shallow cylindrical panels $(0 \leq \widetilde{\alpha} \leq 1)$ exceed the critical stress of the flat panel - rectangular plate $(\widetilde{\alpha}=0)$ (Fig. 3). Therefore, the critical stress of the flat panel - rectangular plate is a lower estimation of critical stresses of perfect shallow cylindrical panels.

\section{Conclusions}

The mathematical description and solution of buckling and post-buckling behaviour of shallow - nearly flat cylindrical panels is presented. The expression (7) for stresses includes both the flat panel and perfect shallow cylindrical panels. The family of shallow shells - almost flat panels is characterized by the sectorial angle. The values of the angle are in the range $0 \leq \alpha \leq \alpha_{s}$. The boundary angle (12) restricts the family of shallow shells. The critical stress of the flat panel - rectangular plate is a lower estimation of critical stresses of perfect shallow cylindrical panels.

Acknowledgements. The presented work was done with the support by the Ministry of Science and Higher Education of Poland.

\section{REFERENCES}

[1] S.P. Timoshenko and J.M. Gere, Theory of Elastic Stability, McGraw-Hill Book Comp. New York, Toronto, London, 1961.

[2] J.W. Hutchinson and W.T. Koiter, "Postbuckling theory", Appl. Mech. Rev. 23, 1353-1356 (1970).

[3] B. Budiansky, "Theory of buckling and post-buckling behaviour of elastic structures", Adv. Appl. Mech. 14, 1-65 (1974).

[4] D.O. Brush and B.O. Almroth, Buckling of Bars, Plates, and Shells, McGraw-Hill Book Comp. New York - Toronto, 1975.

[5] L.H. Donnell, Beams, Plates and Shells, McGraw-Hill Book Comp. New York, 1976.

[6] E.I. Grigoluk and V.V. Kabanov, Stability of Shells, Fiz-Mat-Lit, Moscow (in Russian) 1978.

[7] N. Yamaki, Elastic Stability of Circular Cylindrical Shells. Applied Mathematics and Mechanics, North-Holland, Amsterdam, New York, Oxford, 1984.
[8] D. Bushnell, Computerized Buckling Analysis of Shells, Martinus Nijhoff, Dordrecht, Boston, Lancaster, 1985.

[9] J.G. Simitses, "Buckling and postbuckling of imperfect cylindrical shells", Appl. Mech. Rev. 39, 1517-1524 (1986).

[10] Z.P. Bažant and L. Cedolin, Stability of Structures, Oxford University Press, New York, Oxford, 1991.

[11] G.W. Hunt and E.L. Neto, "Maxwell critical loads for axially loaded cylindrical shells", J. Appl. Mech. T ASME 60, 702-706 (1993).

[12] J.G. Teng, "Buckling of thin shells: Recent advances and trends", Appl. Mech. Rev. 49, 263-274 (1996).

[13] G.J. Lord, A.R. Champneys, and G.W. Hunt, "Computation of localized post buckling in long axially compressed cylindrical shells", Philos Trans. A Math. Phys. Eng. Sci. 355, 2137-2150 (1997).

[14] E. Ventsel and T. Krauthammer, Thin Plates and Shells. Theory, Analysis, and Applications, Marcel Dekker Inc., New York, Basel, 2001.

[15] J.M. Rotter, "Cylindrical shells under axial compression". In: J.G. Teng, J.M. Rotter (Eds.) Buckling of Thin Metal Shells. Spon Press, Taylor and Francis Group, London, New York, 4287 (2004).

[16] N.T.H. Luong and T.H. Tri, "Influence of variable thickness on stability of rectangular plate under compression", Mech. Res. Commun. 32, 139-146 (2005).

[17] K. Magnucki, "Lower critical stress analysis of axially compressed cylindrical shells", Proc. Tenth Intl. Conf. on Civil Structural and Environmental Engineering Computing, B.H.V. Topping (Ed.) Civil-Comp Press, Stirling, Scotland, Paper 52 (2005).

[18] D. Dębowski, K. Magnucki, and M. Malinowski, "Dynamic stability of a metal foam rectangular plate", Steel \& Composite Structures 10, 151-168 (2010).

[19] T. Belica, M. Malinowski, and K. Magnucki, "Dynamic stability of an isotropic metal foam cylindrical shell subjected to external pressure and axial compression", J. Appl. Mech. T ASME 78, Article No 041003 (2011).

[20] D.E. Moulton and A. Goriely, "Circumferential buckling instability of a growing cylindrical tube", J. Mech. Phys. Solids 59, 525-537 (2011).

[21] C. Polat and Y. Calayir, "Nonlinear static and dynamic analysis of shells of revolution", Mech. Res. Commun. 37, 205-209 (2010).

[22] K. Magnucki and P. Jasion, "Analytical description of pre-buckling and buckling states of barrelled shells under radial pressure", Ocean Eng. 58, 217-223 (2013).

[23] W.T. Koiter, "Buckling and post buckling behaviour of a cylindrical panel under axial compression", Reports and Transactions National Aeronautical Research Institute 20, 71-84 (1956) (Nat. Aero. Res. Inst. Report No. S476).

[24] J.M.T. Thompson and G.W. Hunt, A General Theory of Elastic Stability, John Wiley \& Sons, London, New York, Sydney, Toronto, 1973.

[25] E.R. Lancaster, C.R. Calladine, and S.C. Palmer, "Paradoxical buckling behaviour of a thin cylindrical shell under axial compression”, Int. J. Mech. Sci. 42, 843-865 (2000). 